\title{
Particle-hole ring diagrams for fermions in two dimensions
}

\author{
N. Kaiser ${ }^{1}$ \\ Physik-Department T39, Technische Universität München, D-85747 Garching, Germany
}

\begin{abstract}
The set of particle-hole ring diagrams for a many-fermion system in two dimensions is studied. The complex-valued polarization function is derived in detail and shown to be expressible in terms of square-root functions. For a contact-interaction the perturbative contributions to the energy per particle $\bar{E}\left(k_{f}\right)$ are calculated in closed analytical form from third up to twelfth order. The resummation of the particle-hole ring diagrams to all orders is studied and a pronounced dependence on the dimensionless coupling parameter $\alpha$ is found. There is a substantial difference between the complete ring-sum with all exchange-type diagrams included and the standard resummation of the leading $n$-ring diagrams only. The spin factor $S_{n}(g)$ associated to the $n$-th order ring diagrams is derived for arbitrary spin-degeneracy $g$.
\end{abstract}

\section{Introduction}

An important class of diagrams in finite-temperature and many-body calculations is the set of the particle-hole ring diagrams. In gauge theories the ring diagrams play a crucial role since they incorporate screening corrections to the (bare) infinite-range interactions and their inclusion becomes necessary in order to achieve a well behaved perturbative expansion at all [1]. In theories with short-range interactions the particle-hole bubbles provide also important corrections to the effective interaction. An example for this feature is the induced interaction in Landau's theory for normal Fermi-liquids [2,3]. Likewise, the energies and lifetimes of collective excitations of a system (phonons, plasmons etc.) are determined from the poles of the particle-hole polarization propagator.

The particle-hole ring diagrams have also been considered repeatedly in effective field theory approaches to (three-dimensional) many-fermion systems at finite density [4, 5]. Based on a contact-interaction proportional to the s-wave scattering length $a$, the particle-hole ring diagrams contribute at third and higher orders to the systematic low-density expansion of the equation of state $[6,7]$. The respective coefficients occurring in the expansion in powers of $a k_{f}$ are however known only with moderate numerical accuracy. Another feature of the particle-hole ring diagrams with a contact-interaction is that their spin-factor (a polynomial of degree $n$ in $g$ ) varies in a somewhat complicated way with the order $n$ and the spin-degeneracy $g$. For large spin-degeneracy $g$ the particle-hole ring diagrams are actually distinguished as the leading ones in a $1 / g$-expansion (with $a g=$ constant) and their resummation to all orders has been studied in detail in refs. [4, 5]. Moreover, the resummed particle-hole ring diagrams could be used to derive in the Bose limit a non-perturbative result with a non-analytical dependence on the scattering length $a$ and the particle density [4].

The purpose of the present paper is to study in the same way the particle-hole ring diagrams for fermions in two spatial dimensions. The analytical evaluation of fermionic in-medium ladder diagrams in two dimensions together with their resummation to all orders has been presented recently in (the appendix of) ref.[8]. The pertinent complex-valued polarization function $\Pi\left(q_{0},|\mathbf{q}|\right)$ for fermions in two dimensions is rederived in section 2. One finds that both its real and imaginary

\footnotetext{
${ }^{1}$ Email address: nkaiser@ph.tum.de
} 


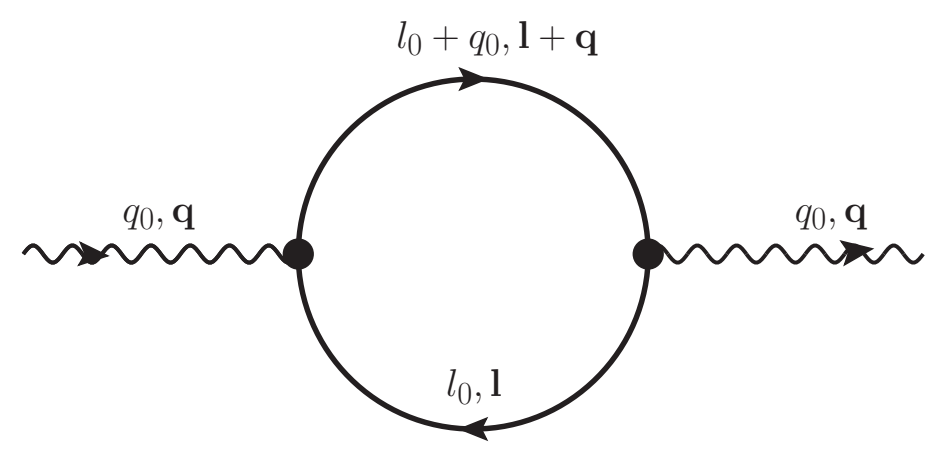

Figure 1: Elementary particle-hole polarization bubble with energy $q_{0}$ and momentum q flowing through it. The wiggly line symbolizes the contact-interaction.

part can be expressed in terms of simple square-root functions of two dimensionless variables. This represents an essential simplification in comparison to the three-dimensional case where logarithmic functions occur in the expression for the real part. Given the polarization function the perturbative contributions to the energy per particle $\bar{E}\left(k_{f}\right)$ as they arise from particle-hole ring diagrams with a contact-interaction are calculated in section 3 . The dependence on the Fermi momentum is $k_{f}^{2} / M$ at each order and, most remarkably, the pertinent numerical coefficients can be given as exact numbers (involving rationals and $\ln 2$ ) from third up to twelfth order. The resummation of the two-dimensional particle-hole ring diagrams to all orders is then studied in section 4 . One finds a dependence on the dimensionless coupling parameter $\alpha$, which is much more pronounced than the dependence on $a k_{f}$ in the three-dimensional case. Moreover, the complete ring-sum with all exchange-type diagrams included differs substantially from the resummation of the leading $n$-ring diagrams only. In the appendix a derivation of the formula $S_{n}(g)=(g-1)^{n}+$ $(-1)^{n}\left(g^{2}-1\right)$ for the general spin-factor of $n$-th order particle-hole ring diagrams is presented.

It is hoped that the results of the present paper will find applications in special condensed matter systems (such as two-dimensional graphene [9] or strongly interacting two-dimensional Fermi gases $[10,11])$.

\section{Polarization function}

The basic quantity for treating the particle-hole ring diagrams is the complex-valued polarization function $\Pi\left(q_{0},|\mathbf{q}|\right)$, depending on energy $q_{0}$ and momentum $|\mathbf{q}|$. For non-relativistic fermions in three dimensions its explicit derivation can be found e.g. in the textbook [12]. The expression for the polarizability of the two-dimensional electron gas has been given long ago by Stern in ref.[13]. Nevertheless, it is instructive to rederive the result for the two-dimensional polarization function by presenting some details of the calculation. In this work we consider spin- $1 / 2$ fermions of mass $M$ in two dimensions and introduce a two-body contact-interaction with coupling constant $C_{0}=2 \pi \alpha / M$, where $\alpha$ is a dimensionless parameter. When including this coupling constant and a factor -2 from the sum over spins and the closed fermion loop in Fig. 1, the two-dimensional polarization function reads:

$$
\begin{aligned}
\Pi\left(q_{0},|\mathbf{q}|\right)= & -\frac{4 \pi i \alpha}{M} \int \frac{d l_{0}}{2 \pi} \int \frac{d^{2} \mathbf{l}}{(2 \pi)^{2}}\left\{\frac{i}{l_{0}-\mathbf{l}^{2} / 2 M+i \epsilon}-2 \pi \delta\left(l_{0}-\mathbf{l}^{2} / 2 M\right) \theta\left(k_{f}-|\mathbf{l}|\right)\right\} \\
& \times\left\{\frac{i}{l_{0}+q_{0}-(\mathbf{l}+\mathbf{q})^{2} / 2 M+i \epsilon}-2 \pi \delta\left(l_{0}+q_{0}-(\mathbf{l}+\mathbf{q})^{2} / 2 M\right) \theta\left(k_{f}-|\mathbf{l}+\mathbf{q}|\right)\right\}
\end{aligned}
$$


Here, each factor in the curly brackets is a non-relativistic particle-hole propagator which has been rewritten identically as the sum of a vacuum-propagator and a medium-insertion. The Fermi momentum $k_{f}$ appearing in the step-functions $\theta(\ldots)$ is related to the particle density (per area) by $\rho_{2}=k_{f}^{2} / 2 \pi$. By shifting the integration variable $l_{0} \rightarrow l_{0}-q_{0} / 2$, one deduces immediately that $\Pi\left(q_{0},|\mathbf{q}|\right)$ is an even function of the energy $q_{0}$. Therefore, one can assume $q_{0} \geq 0$ in the following. Next, the momentum-space integrals are evaluated for all four terms in eq.(1). The term with no step-function gives zero upon $l_{0}$-integration with residue calculus, since both poles lie in the lower $l_{0}$ half-plane. The two contributions with one medium-insertion lead to a real part of the form:

$$
\operatorname{Re} \Pi\left(q_{0},|\mathbf{q}|\right)=4 \pi \alpha f \frac{d^{2} \mathbf{l}}{(2 \pi)^{2}}\left\{\frac{\theta\left(k_{f}-|\mathbf{l}|\right)}{\mathbf{l} \cdot \mathbf{q}+\mathbf{q}^{2} / 2-M q_{0}}+\left(q_{0} \rightarrow-q_{0}\right)\right\},
$$

where we have substituted $\mathbf{l} \rightarrow-\mathbf{l}-\mathbf{q}$ in the second contribution. It is convenient to introduce dimensionless variables $(x, \nu)$ by setting $|\mathbf{q}|=2 x k_{f}$ and $q_{0}=2 \nu x k_{f}^{2} / M$, where the extra factor $x$ in the parametrization of $q_{0}$ simplifies all following formulas. The remaining principal-value integral is readily solved by using planar polar coordinates:

$$
\begin{aligned}
& \frac{\alpha}{2 \pi x} \int_{0}^{1} d \lambda f_{0}^{\pi} d \varphi(x-\nu+\sqrt{\lambda} \cos \varphi)^{-1}=\frac{\alpha}{2 x} \operatorname{sign}(x-\nu) \operatorname{Re} \int_{0}^{1} d \lambda\left[(x-\nu)^{2}-\lambda\right]^{-1 / 2} \\
& =\frac{\alpha}{x} \operatorname{sign}(x-\nu) \operatorname{Re}\left\{|x-\nu|-\sqrt{(x-\nu)^{2}-1}\right\} .
\end{aligned}
$$

The second term in eq.(2) is included by adding the same expression with $\nu \rightarrow-\nu$. Scaling out the parameter $\alpha$, one gets finally for the real part of the two-dimensional polarization bubble $\operatorname{Re} \Pi\left(q_{0},|\mathbf{q}|\right)=\alpha R(\nu, x)$ with the function:

$$
R(\nu, x)=2-\frac{1}{x} \operatorname{Re}\left\{\operatorname{sign}(x+\nu) \sqrt{(x+\nu)^{2}-1}+\operatorname{sign}(x-\nu) \sqrt{(x-\nu)^{2}-1}\right\} .
$$

The imaginary part of the two-dimensional polarization function has the representation:

$$
\begin{array}{r}
\operatorname{Im} \Pi\left(q_{0},|\mathbf{q}|\right)=\alpha \int d^{2} \mathbf{l} \delta\left(M q_{0}-\mathbf{l} \cdot \mathbf{q}\right)\left\{\theta\left(k_{f}-|\mathbf{l}-\mathbf{q} / 2|\right)+\theta\left(k_{f}-|\mathbf{l}+\mathbf{q} / 2|\right)\right. \\
\left.-2 \theta\left(k_{f}-|\mathbf{l}-\mathbf{q} / 2|\right) \theta\left(k_{f}-|\mathbf{l}+\mathbf{q} / 2|\right)\right\},
\end{array}
$$

where the shift $\mathbf{l} \rightarrow \mathbf{l}-\mathbf{q} / 2$ has been made. By analyzing for the first term in eq.(5) the intersection of a straight line (as imposed by $\delta\left(M q_{0}-\mathbf{l} \cdot \mathbf{q}\right)$ ) and a shifted circular disc in the l-plane, one finds that it contributes to the imaginary part with the term: $\alpha x^{-1} \sqrt{1-(x-\nu)^{2}}$ for $x-1<\nu<x+1$. Via the substitution $\mathbf{l} \rightarrow-\mathbf{l}$ the contribution of the second term in eq.(5) is obtained by altering the sign of $\nu$ and thus it becomes: $\alpha x^{-1} \sqrt{1-(x+\nu)^{2}}$ for $-x-1<\nu<1-x$. Since only positive $\nu$ need to be considered the latter condition is equivalent to $0<\nu<1-x$. In order that the third term in eq.(5) contributes at all, the two shifted Fermi discs must overlap, which requires $0<x<1$. In this case the third term subtracts twice the second contribution in the region $0<\nu<1-x$, and thus effectively reverses the sign of that second contribution. Putting all three pieces together and scaling out $\alpha$ again, one obtains for the imaginary part of the two-dimensional polarization bubble $\operatorname{Im} \Pi\left(q_{0},|\mathbf{q}|\right)=\alpha I(\nu, x)$ with the function:

$$
\begin{aligned}
& I(\nu, x)=\frac{1}{x} \sqrt{1-(x-\nu)^{2}}, \text { for }|x-1|<\nu<x+1, \\
& I(\nu, x)=\frac{1}{x}\left\{\sqrt{1-(x-\nu)^{2}}-\sqrt{1-(x+\nu)^{2}}\right\}, \text { for } \quad 0<\nu<1-x .
\end{aligned}
$$




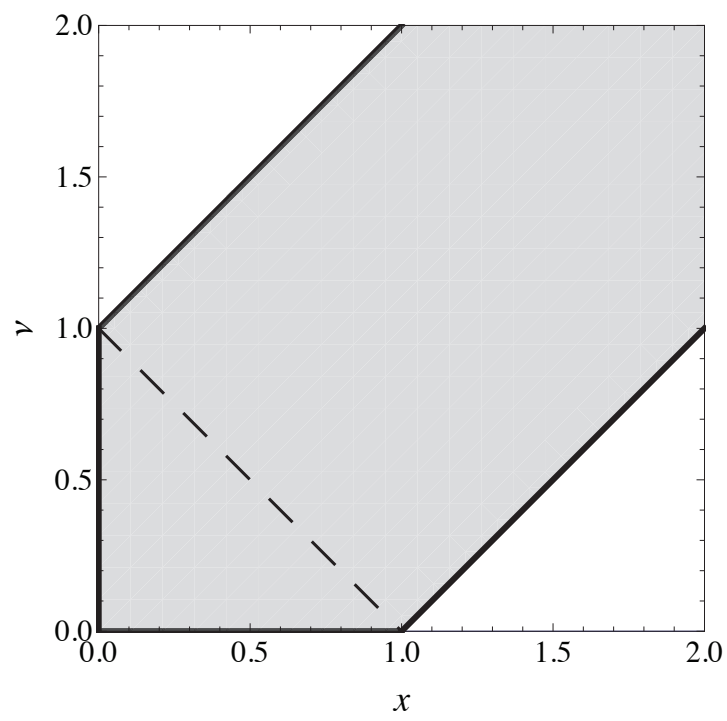

Figure 2: Integration region in the $x \nu$ quarter-plane in between the two straight lines $\nu=x-1$ and $\nu=x+1$. The functions $R(\nu, x)$ and $I(\nu, x)$ have different forms below and above the dashed line $\nu=1-x$.

It is interesting to note that the imaginary part of three-dimensional polarization function is of similar structure $[4,5,12]$. The parameter $\alpha$ gets replaced by $a k_{f} / 2$ and the individual terms in eq.(6) come without square-roots. The real part of the three-dimensional polarization function is composed of logarithms: $\ln [(1+x+\nu) /|1-x-\nu|]$ and $\ln [|1+x-\nu| /|1-x+\nu|]$. One should also note that the Lindhard functions of a Fermi gas in one, two, and three dimensions have been studied extensively and compared with each other in ref.[14]. After translating the dimensionless variables $(\nu, x)$ the present results for $R(\nu, x)$ and $I(\nu, x)$ written in eqs. $(4,6)$ agree exactly with the earlier calculations in refs. $[13,14]$.

Fig. 2 shows the support of the function $I(\nu, x)$ in the (positive) $x \nu$-plane. The corresponding region lies in between the two straight lines $\nu=x-1$ and $\nu=x+1$. When crossing the (dashed) line $\nu=1-x$, the function $I(\nu, x)$ changes its form and it vanishes along the circumference (with the exception of the line-segment $x=0,0<\nu<1)$. We anticipate that the real-valued integrand relevant for calculating the interaction energy density from particle-hole ring diagrams involves a proportionality factor $I(\nu, x)$. Exploiting this property, the function $R(\nu, x)$ can be restricted to the support of $I(\nu, x)$. Inspection of eq.(4) shows that the last term can be dropped, since its radicand $[\nu-(x+1)][\nu-(x-1)]$ is negative. Furthermore, in the region $0<\nu<1-x$ the other radicand $[\nu+(x+1)][\nu-(1-x)]$ becomes also negative. Altogether, the function $R(\nu, x)$ simplifies considerably to:

$$
\begin{aligned}
& R(\nu, x)=2-\frac{1}{x} \sqrt{(x+\nu)^{2}-1}, \quad \text { for } \quad|x-1|<\nu<x+1, \\
& R(\nu, x)=2, \quad \text { for } \quad 0<\nu<1-x, \quad 0<x<1,
\end{aligned}
$$

inside the relevant integration region.

\section{$3 \quad$ Energy per particle and perturbative results}

In this section we calculate the energy per particle $\bar{E}\left(k_{f}\right)$ as it arises from ring diagrams with a contact-interaction. For orientation we note that the free Fermi gas energy in two dimensions is 
$\bar{E}\left(k_{f}\right)^{(0)}=k_{f}^{2} / 4 M$. Likewise, the first-order Hartree-Fock contribution from a contact-coupling $C_{0}=2 \pi \alpha / M$ is given by $\bar{E}\left(k_{f}\right)^{(1)}=-\alpha k_{f}^{2} / 4 M$. Obviously, the energy density arising from the leading $n$-ring diagram (obtained by connecting $n$ polarization bubbles in Fig. 1 to a closed ring) is determined by the $n$-th power of the polarization function $\Pi\left(q_{0},|\mathbf{q}|\right)$. With inclusion of the symmetry factor $1 / 2 n$ the corresponding energy density reads:

$$
\frac{k_{f}^{2}}{2 \pi} \bar{E}\left(k_{f}\right)_{\text {lead }}^{n-\text { ring }}=\frac{i}{2 n} \int \frac{d q_{0}}{2 \pi} \int \frac{d^{2} \mathbf{q}}{(2 \pi)^{2}}\left[\Pi\left(q_{0},|\mathbf{q}|\right)\right]^{n} .
$$

After transforming to the dimensionless variables $(x, \nu)$ one obtains the following double-integral representation for the contribution of $n$-ring diagrams to the energy per particle:

$$
\bar{E}\left(k_{f}\right)^{n-\text { ring }}=\frac{4 k_{f}^{2}}{\pi M} \frac{\alpha^{n}}{n} \frac{S_{n}}{2^{n}} \int_{0}^{\infty} d x x^{2} \int_{\nu_{\min }}^{x+1} d \nu \operatorname{Im}[R(\nu, x)-i I(\nu, x)]^{n},
$$

with $\nu_{\min }=\max (0, x-1)$. In addition to the leading $n$-ring diagram with spin-factor $2^{n}$, there are various exchange-type ring-diagrams due to the Pauli exclusion-principle. For a contactinteraction these can all be summed together by means of the total spin-factor $S_{n}=1+3(-1)^{n}$. The corresponding sequence is $S_{n}=(-2,4,-2,4, \ldots)$ [5]. In the appendix an explicit derivation of the formula $S_{n}=1+3(-1)^{n}$, using multiple spin-traces, is given. The generalization to arbitrary spin-degeneracy $g \geq 2$ is also presented there.

Based on the representation in eq.(9) the respective double-integrals have been studied in detail by analytical and numerical methods. From third up to twelfth order analytical results in closed form could be obtained for the contributions to the energy per particle $\bar{E}\left(k_{f}\right)$. We list them one after the other:

$n=3$ : the integrand $I\left(I^{2}-3 R^{2}\right)$ leads to the 3-ring contribution:

$$
\bar{E}\left(k_{f}\right)^{3-\text { ring }}=\frac{k_{f}^{2}}{4 M} \alpha^{3}(2 \ln 2-1),
$$

$n=4$ : the integrand $4 I R\left(I^{2}-R^{2}\right)$ leads to the 4-ring contribution:

$$
\bar{E}\left(k_{f}\right)^{4-\text { ring }}=\frac{k_{f}^{2}}{M} \alpha^{4}(2-3 \ln 2),
$$

$n=5$ : the integrand $I\left(10 I^{2} R^{2}-5 R^{4}-I^{4}\right)$ leads to the 5-ring contribution:

$$
\bar{E}\left(k_{f}\right)^{5-\text { ring }}=\frac{k_{f}^{2}}{M} \alpha^{5}\left(4 \ln 2-\frac{11}{4}\right)
$$

$n=6$ : the integrand $2 I R\left(3 R^{2}-I^{2}\right)\left(3 I^{2}-R^{2}\right)$ leads to the 6 -ring contribution:

$$
\bar{E}\left(k_{f}\right)^{6-\text { ring }}=\frac{k_{f}^{2}}{M} \alpha^{6}\left(\frac{83}{6}-20 \ln 2\right),
$$

$n=7$ : the integrand $I^{7}+7 I R^{2}\left(5 I^{2} R^{2}-3 I^{4}-R^{4}\right)$ leads to the 7 -ring contribution:

$$
\bar{E}\left(k_{f}\right)^{7-\text { ring }}=\frac{k_{f}^{2}}{M} \alpha^{7}\left(24 \ln 2-\frac{133}{8}\right),
$$

$n=8$ : the integrand $8 I R\left(I^{2}-R^{2}\right)\left(I^{4}-6 I^{2} R^{2}+R^{4}\right)$ leads to the 8-ring contribution:

$$
\bar{E}\left(k_{f}\right)^{8-\text { ring }}=\frac{k_{f}^{2}}{M} \alpha^{8}\left(\frac{4657}{60}-112 \ln 2\right),
$$


$n=9$ : the integrand $I\left(I^{2}-3 R^{2}\right)\left(33 I^{4} R^{2}-I^{6}-27 I^{2} R^{4}+3 R^{6}\right)$ leads to the 9-ring contribution:

$$
\bar{E}\left(k_{f}\right)^{9-\text { ring }}=\frac{k_{f}^{2}}{M} \alpha^{9}\left(128 \ln 2-\frac{5323}{60}\right),
$$

$n=10$ : the integrand $2 I R\left(10 I^{2} R^{2}-5 I^{4}-R^{4}\right)\left(I^{4}-10 I^{2} R^{2}+5 R^{4}\right)$ leads to the 10 -ring contribution:

$$
\bar{E}\left(k_{f}\right)^{10-\text { ring }}=\frac{k_{f}^{2}}{M} \alpha^{10}\left(\frac{27947}{70}-576 \ln 2\right)
$$

$n=11$ : the integrand $I^{11}+11 I R^{2}\left(30 I^{6} R^{2}-5 I^{8}-42 I^{4} R^{4}+15 I^{2} R^{6}-R^{8}\right)$ leads to the 11-ring contribution:

$$
\bar{E}\left(k_{f}\right)^{11-\text { ring }}=\frac{k_{f}^{2}}{M} \alpha^{11}\left(640 \ln 2-\frac{149053}{336}\right),
$$

$n=12$ : the integrand $4 I R\left(I^{2}-R^{2}\right)\left(I^{2}-3 R^{2}\right)\left(3 I^{2}-R^{2}\right)\left(I^{4}-14 I^{2} R^{2}+R^{4}\right)$ leads to the 12 -ring contribution:

$$
\bar{E}\left(k_{f}\right)^{12-\text { ring }}=\frac{k_{f}^{2}}{M} \alpha^{12}\left(\frac{4918777}{2520}-2816 \ln 2\right) .
$$

Following the pattern of numerical coefficients in eqs.(10-19) one conjectures for the $n$-ring contribution:

$$
\bar{E}\left(k_{f}\right)^{n-\text { ring }}=\frac{k_{f}^{2}}{M}(-\alpha)^{n}\left\{\zeta_{n}-(n-1) 2^{n-6}\left[3+(-1)^{n}\right] \ln 2\right\},
$$

with $\zeta_{n}$ a positive rational number. In the course of the calculation the following observations have been made. The contribution to the double-integral in eq.(9) from the (triangular) region $0<\nu<1-x$ (see Fig. 2) can be solved analytically, with a result equal to $\pi$ times a rational linear combination of 1 and $\ln 2$. The integral over the (rectangular) region $|x-1|<\nu<x+1$ is numerically extremely close to a rational multiple of $\pi$. The corresponding values in the case $n=3$ are $\pi(7-12 \ln 2) / 8$ and $-\pi / 8$. In the cases $n=4,5,6,7$ the numerical integral over the rectangular region $|x-1|<\nu<x+1$ vanishes with an accuracy $10^{-10}$ or better, ${ }^{2}$ while for $n=8$ it has the value $-256 \pi / 15$. For $n \geq 9$ the integrals over both regions develop divergences which cancel in the sum. It is then advantageous to map the entire integration region onto a unit square $0<x, y<1$ via substitutions: $\nu=(1-x) y, \nu=1-x+2 x y$, and $\nu=x-1+2 y, x \rightarrow 1 / x$. In order to illustrate the available precision we note that the large integers 4657, 5323, 27947, 149053 and 4918777 in eqs.(15-19) are reproduced with a relative accuracy of $10^{-13}$ and better. The list of numerical coefficients $c_{n}$ for the $n$-ring contribution to the energy per particle $\bar{E}\left(k_{f}\right)^{n-\text { ring }}=c_{n} \alpha^{n} k_{f}^{2} / M$ is continued up to $n=30$ in Table 1 . One observes that the sequences of positive and negative coefficients $c_{n}$ decrease both slowly in magnitude.

Let us also give an alternative representation for the ring energy $\bar{E}\left(k_{f}\right)^{n \text {-ring }}$ at $n$-th order. When following the method described in the text book by Gross, Runge and Heinonen [15] and evaluating the key quantity defined in eq.(22.15) in two dimensions, one obtains:

$$
\bar{E}\left(k_{f}\right)^{n-\text { ring }}=-\frac{4 k_{f}^{2}}{\pi M} \frac{S_{n} \alpha^{n}}{n} \int_{0}^{\infty} d x x^{2} \int_{0}^{\infty} d y[K(x, y)]^{n},
$$

with the (two-dimensional) euclidean polarization function:

$$
K(x, y)=1-\frac{1}{\sqrt{2} x} \sqrt{x^{2}-y^{2}-1+\sqrt{\left(1+x^{2}+y^{2}\right)^{2}-4 x^{2}}}=1-\frac{1}{x}\left|\operatorname{Re} \sqrt{(x+i y)^{2}-1}\right| .
$$

\footnotetext{
${ }^{2}$ For the analytical and numerical treatment of the integrals is it occasionally useful to introduce new coordinates by $x=(\xi+\eta) / 2, \nu=(\xi-\eta) / 2$. The integration region in the $\xi \eta$-plane consists then of a triangle $-\xi<\eta<\xi$, $0<\xi<1$ and a semi-infinite strip $-1<\eta<1, \xi>1$.
} 


\begin{tabular}{|c|ccccccc|}
\hline$n$ & 3 & 4 & 5 & 6 & 7 & 8 & 9 \\
\hline $100 c_{n}$ & 9.657359 & -7.944154 & 2.258872 & -2.961028 & 1.053233 & -1.581756 & 0.617245 \\
\hline$n$ & 10 & 11 & 12 & 13 & 14 & 15 & 16 \\
\hline $100 c_{n}$ & -0.991886 & 0.407651 & -0.682554 & 0.290058 & -0.499344 & 0.217243 & -0.381589 \\
\hline$n$ & 17 & 18 & 19 & 20 & 21 & 22 & 23 \\
\hline $100 c_{n}$ & 0.168933 & -0.301301 & 0.135201 & -0.244050 & 0.110695 & -0.201763 & 0.092323 \\
\hline$n$ & 24 & 25 & 26 & 27 & 28 & 29 & 30 \\
\hline $100 c_{n}$ & -0.169628 & 0.078189 & -0.144627 & 0.067079 & -0.124789 & 0.058186 & -0.108781 \\
\hline
\end{tabular}

Table 1: Numerical coefficients $c_{n}$ of the energy per particle $\bar{E}\left(k_{f}\right)^{n-\text { ring }}=c_{n} \alpha^{n} k_{f}^{2} / M$ arising from the $n$-th order particle-hole ring diagrams with a contact-interaction $2 \pi \alpha / M$.

Note that the absolute magnitude in the short form removes the sign-ambiguity of the complex square-root. The function $K(x, y)$ takes on values in the range $0<K(x, y)<1$ and its asymptotic behavior in the $x y$ quarter plane is:

$$
K(r \cos \varphi, r \sin \varphi)=\frac{1}{2 r^{2}}+\frac{1-4 \sin ^{2} \varphi}{8 r^{4}}+\mathcal{O}\left(r^{-6}\right) .
$$

Using the representation in eq.(21) all previous results collected in eqs.(10-19) could be reproduced with very high numerical accuracy.

\section{Resummation to all orders}

After the perturbative analysis of the two-dimensional ring diagrams with a contact-interaction we study now their resummation to all orders. Returning to eqs. $(8,9)$ one sees that the resummation to all orders is provided by an arctangent-function with two subtractions. The subtraction terms (linear and quadratic in $\alpha$ ) introduce divergences (quadratic and logarithmic in the upper limit of the $x$-integration) which get canceled by the integral over the arctangent-function. In order to avoid such numerically delicate cancellations one better represents the derivative of $\bar{E}\left(k_{f}\right)$ with respect to $\alpha$ in terms of a convergent integral over a rational expression and integrates back in $\alpha$ :

$$
\begin{aligned}
\bar{E}\left(k_{f}\right)_{\text {lead }}= & \frac{4 k_{f}^{2}}{\pi M} \int_{0}^{\alpha} d z z^{2} \int_{0}^{\infty} d x x^{2} \int_{\nu_{\min }}^{x+1} d \nu I(\nu, x) \\
& \times \frac{I(\nu, x)^{2}-3 R(\nu, x)^{2}+2 z R(\nu, x)\left[R(\nu, x)^{2}+I(\nu, x)^{2}\right]}{[1-z R(\nu, x)]^{2}+z^{2} I(\nu, x)^{2}}=\frac{k_{f}^{2} \alpha^{3}}{4 M} G(\alpha) .
\end{aligned}
$$

Here, we are considering first the sum of the leading $n$-ring diagrams with spin-factors $2^{n}$. The dimensionless function $G(\alpha)$ is defined by scaling out the free Fermi gas energy $k_{f}^{2} / 4 M$ and the leading power $\alpha^{3}$. Its non-vanishing value at $\alpha=0$ is: $G(0)=4-8 \ln 2=-1.54518$.

Fig. 3 shows the behavior of the function $G(\alpha)$ in the range $-10<\alpha<10$ of the coupling parameter $\alpha$. One observes a sharp spike in the vicinity of $\alpha=1 / 2$. The function $G(\alpha)$ crosses zero twice, at $\alpha \simeq-6.33$ and $\alpha \simeq 1.11$, and in the region $|\alpha|>2$ its variation becomes rather weak. A detailed numerical study at large positive and negative parameter values reveals that the asymptotic behavior of $G(\alpha)$ is given by:

$$
G(\alpha) \simeq \frac{2}{3}+\frac{2 \ln |4 \alpha|-5 / 2}{\alpha} .
$$




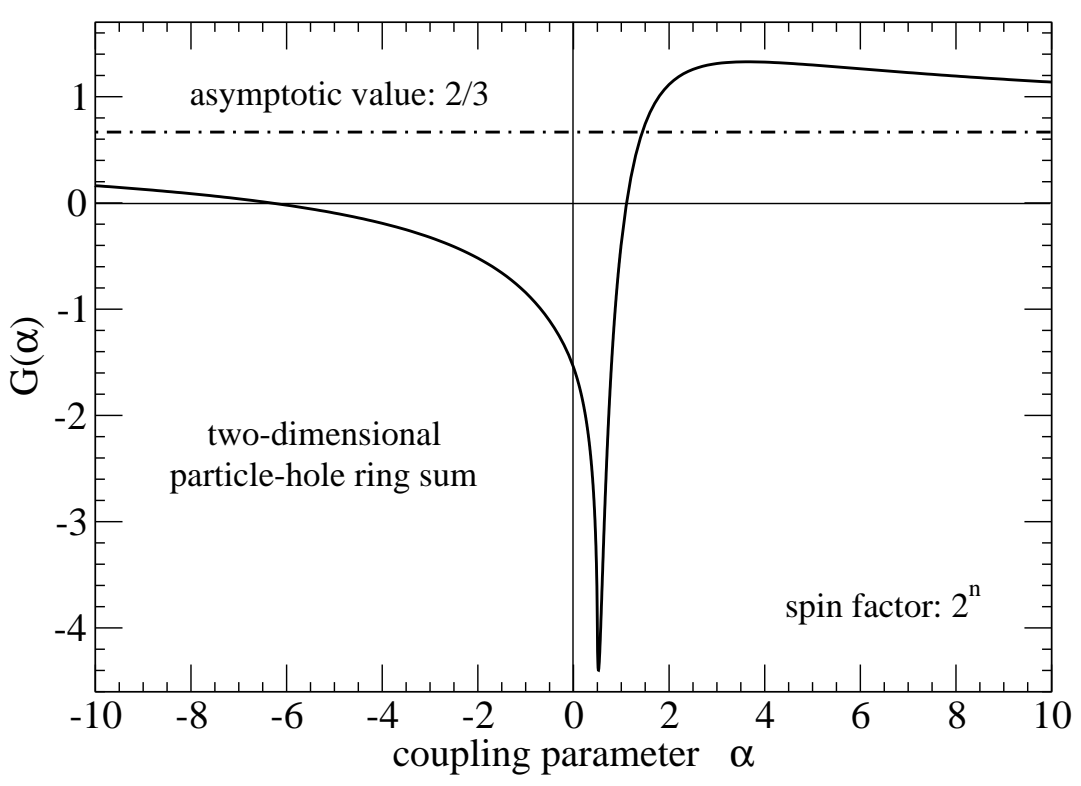

Figure 3: The function $G(\alpha)$ resulting from the resummed particle-hole ring diagrams.

This functional form has been found through a fit to the numerical data at large $|\alpha|$. The behavior of the function $G(\alpha)$ in the region $0<\alpha<1$ around the spike is shown with magnification in Fig. 4. One makes the interesting numerical observation that $G(1 / 4)=-2$ and $G(1 / 2)=-4$ with an accuracy of $10^{-9}$. Furthermore, one sees that $G(\alpha)$ decreases further and reaches its minimum value of about -4.40 at the parameter value $\alpha \simeq 0.523$. One also recognizes from the magnified representation in Fig. 4 that the behavior of $G(\alpha)$ around the "spike" is actually completely smooth. All these observed properties together with the results in eqs.(10-19) lead to the suggestion that the function $G(\alpha)$ could possibly be expressed through some decent analytical formula (involving presumably elliptic or hypergeometric functions). We have however not succeeded in finding such an analytical formula.

Next, we consider the complete ring-sum with inclusion of all exchange-type diagrams. Based on the spin-factor $S_{n}=1+3(-1)^{n}$ at $n$-th order (see eq.(9)) one obtains the following representation of the resummed energy per particle:

$$
\begin{aligned}
\bar{E}\left(k_{f}\right)= & \frac{4 k_{f}^{2}}{\pi M} \int_{0}^{\infty} d x x^{2} \int_{\nu_{\min }}^{x+1} d \nu\{\alpha I(\nu, x)[\alpha R(\nu, x)-1] \\
& \left.+\arctan \frac{\alpha I(\nu, x)}{\alpha R(\nu, x)-2}+3 \arctan \frac{\alpha I(\nu, x)}{\alpha R(\nu, x)+2}\right\}=\frac{k_{f}^{2} \alpha^{3}}{4 M} F(\alpha) .
\end{aligned}
$$

The function $F(\alpha)$ defined by scaling out the free Fermi gas energy $k_{f}^{2} / 4 M$ and the leading power $\alpha^{3}$ is not independent. The relation to the function $G(\alpha)$ introduced before in eq.(24) reads:

$$
F(\alpha)=\frac{1}{8}\{G(\alpha / 2)-3 G(-\alpha / 2)\} .
$$

Fig. 5 shows the behavior of the function $F(\alpha)$ in the range $-10<\alpha<10$ of the coupling parameter $\alpha$. One observes now two peaks located at $\alpha \simeq \pm 1$, which simply arise from the minima of $G( \pm \alpha / 2)$ (see Figs. 3,4). The value at $\alpha=0$ is $F(0)=2 \ln 2-1=0.386294$ and asymptotically $F( \pm \infty)=-1 / 6$ is reached. In order to reveal the strong parameter dependence in the region $-4<\alpha<4$ it is instrumental to scale out the trivial factor $\alpha^{3}$ in eq.(26). When 


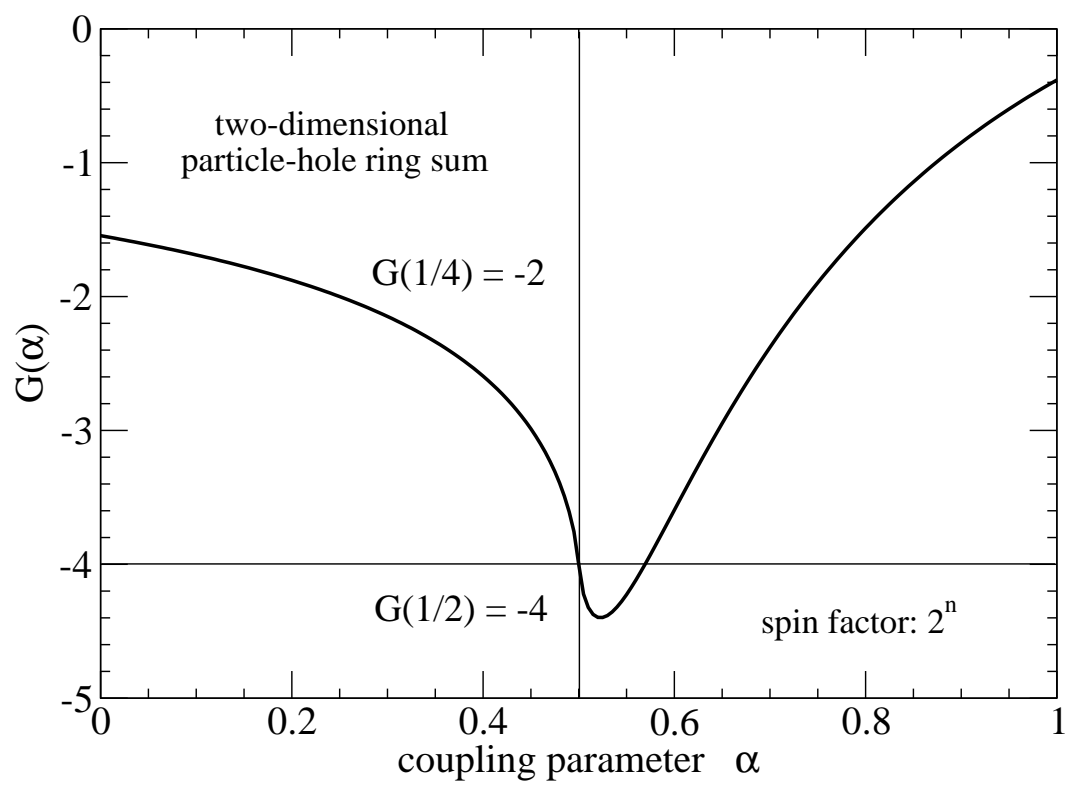

Figure 4: The function $G(\alpha)$ magnified in the region $0<\alpha<1$.

scanning through from large negative to large positive values of coupling constant $\alpha$, the energy per particle $\bar{E}\left(k_{f}\right)$ changes its sign four times. This is a rather striking parameter dependence. Altogether, the main finding of the present work is that complete ring-sum with all exchange-type diagrams included leads to a quantitatively and qualitatively different result for the energy per particle $\bar{E}\left(k_{f}\right)$ in comparison to the standard resummation of the leading $n$-ring diagrams.

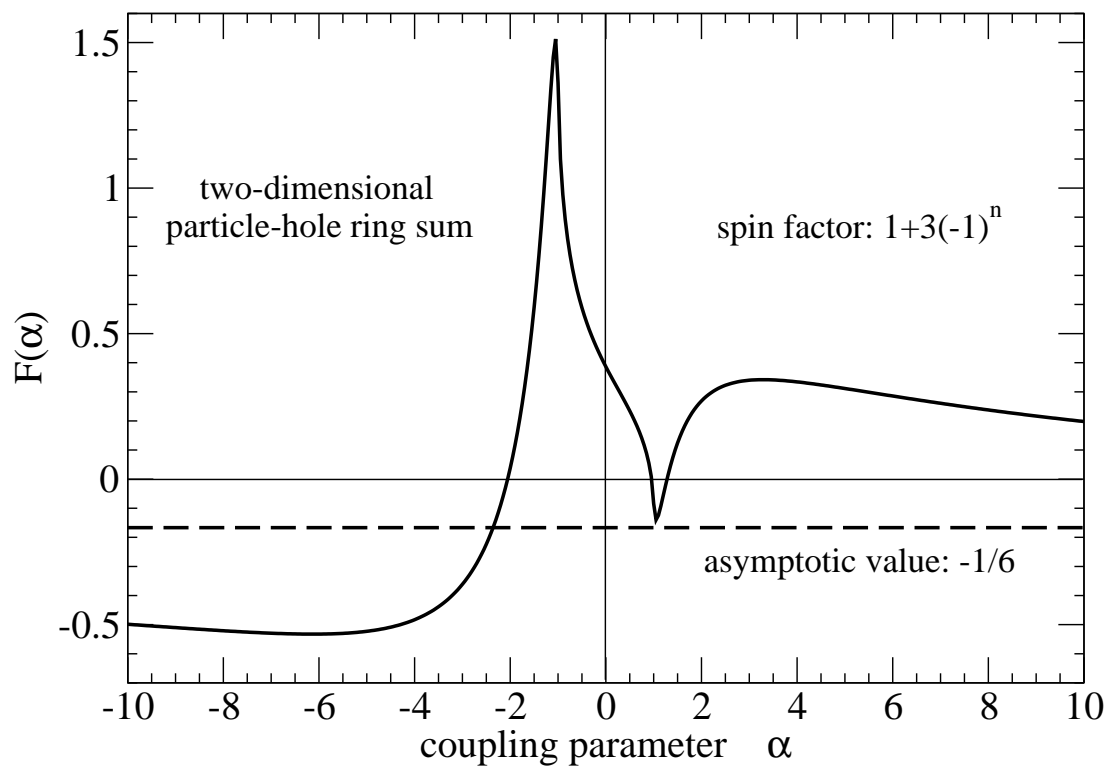

Figure 5: The function $F(\alpha)$ resulting from the resummed particle-hole ring diagrams.

It is also interesting to compare with the analogous results for the resummed ring diagrams in three dimensions $[4,5]$. Using the product $a k_{f}$ of scattering length $a$ and Fermi momentum $k_{f}$ as a dimensionless coupling strength ${ }^{3}$ and appropriate polarization functions $I_{3}(\nu, x)$ and $R_{3}(\nu, x)$,

\footnotetext{
${ }^{3}$ The sign-convention is chosen such that $a>0$ corresponds to attraction.
} 


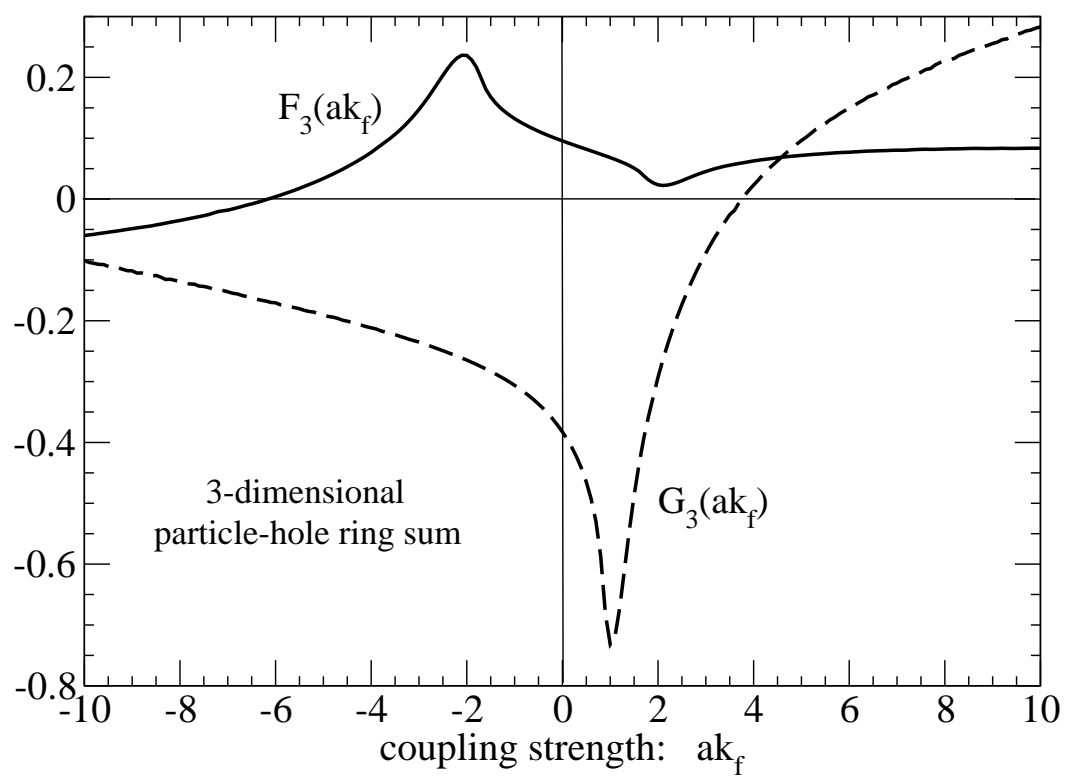

Figure 6: The functions $G_{3}\left(a k_{f}\right)$ and $F_{3}\left(a k_{f}\right)$ resulting from the resummed particle-hole ring diagrams with a contact-interaction $4 \pi a / M$ in three dimensions.

the only difference to eqs. $(24,26)$ is an additional factor $3 x$. The functions $G_{3}\left(a k_{f}\right)$ and $F_{3}\left(a k_{f}\right)=$ $\left[G_{3}\left(a k_{f} / 2\right)-3 G_{3}\left(-a k_{f} / 2\right)\right] / 8$ are defined in the same way by dividing the resummed energy per particle $\bar{E}\left(k_{f}\right)$ by the three-dimensional free Fermi gas energy $3 k_{f}^{2} / 10 M$ and the leading power $\left(a k_{f}\right)^{3}$. These two functions are shown by the dashed and full line in Fig. 6 for $-10<a k_{f}<10$. One observes a behavior similar to the one in Figs. 3,5, however with a less pronounced dependence on the dimensionless coupling strength $a k_{f}$. The function $G_{3}\left(a k_{f}\right)$ reaches its minimum of -0.732 at $a k_{f} \simeq 1.0$ and it crosses zero at $a k_{f} \simeq 3.75$. The asymptotic behavior of $G_{3}\left(a k_{f}\right)$ is not obvious from Fig. 6 and also very difficult to extract by numerical methods [4]. One can conclude that the two-dimensional particle-hole ring diagrams with their multitude of analytical results represent a rather special case.

\section{$5 \quad$ Summary and conclusions}

In this work we have studied the particle-hole ring-diagrams for a many-fermion in two dimensions. Based on a contact-interaction the perturbative contributions to the energy per particle $\bar{E}\left(k_{f}\right)$ could be given in closed analytical from third up to twelfth order. The resummation to all orders exhibits a pronounced dependence on the dimensional coupling parameter $\alpha$. A particular finding of the present work has been that the complete ring-sum with all exchange-type diagrams included (corresponding to spin-factors $1+3(-1)^{n}$ ) leads to a significantly different result for the energy per particle than the standard resummation of the leading $n$-ring diagrams (with spin-factors $2^{n}$ ).

\section{Appendix: Calculation of general spin-factor}

In this appendix we present a derivation of the general spin-factor associated to the particlehole ring diagrams at $n$-th order. We start with the simplest case of spin- $1 / 2$ fermions, i.e. spin-degeneracy $g=2$. While the leading $n$-ring diagram has the obvious spin-factor $g^{n}=2^{n}$, 
the problem consists in determining this factor for all the exchange-type diagrams. For a fourfermion contact-interaction the exchange-type diagrams required by the Pauli exclusion-principle are automatically taken into account if the antisymmetrized form of the contact-interaction is used:

$$
\mathbf{1}-\frac{1}{2}\left(\mathbf{1}+\vec{\sigma}_{1} \cdot \vec{\sigma}_{2}\right)=\frac{1}{2}\left(\mathbf{1}-\vec{\sigma}_{1} \cdot \vec{\sigma}_{2}\right)
$$

as obtained by subtracting the spin-exchange operator. The resulting operator $\left(\mathbf{1}-\vec{\sigma}_{1} \cdot \vec{\sigma}_{2}\right) / 2$ acts in the product spin-space of particle 1 and particle 2 . The total spin-factor $S_{n}$ of the $n$-ring diagrams is now given by the trace of the product of $n$ such operators multiplied in cyclic order of their particle indices. The starting expression reads:

$$
\begin{aligned}
S_{n} & =\frac{1}{2^{n}} \operatorname{tr}_{1} \operatorname{tr}_{2} \ldots \operatorname{tr}_{n}\left\{\left(\mathbf{1}-\vec{\sigma}_{n} \cdot \vec{\sigma}_{1}\right)\left(\mathbf{1}-\vec{\sigma}_{1} \cdot \vec{\sigma}_{2}\right) \ldots\left(\mathbf{1}-\vec{\sigma}_{n-1} \cdot \vec{\sigma}_{n}\right)\right\} \\
& =\frac{2}{2^{n}} \operatorname{tr}_{2} \ldots \operatorname{tr}_{n}\left\{\left(\mathbf{1}+\vec{\sigma}_{n} \cdot \vec{\sigma}_{2}\right) \ldots\left(\mathbf{1}-\vec{\sigma}_{n-1} \cdot \vec{\sigma}_{n}\right)\right\},
\end{aligned}
$$

and we have performed in the second line the trace in the spin-space of particle 1 . Note the sign change in front of the spin-spin operator $\vec{\sigma}_{n} \cdot \vec{\sigma}_{2}$. After taking $n-1$ spin-traces one arrives at:

$$
S_{n}=\frac{2^{n-1}}{2^{n}} \operatorname{tr}_{n}\left\{\mathbf{1}+(-1)^{n} \vec{\sigma}_{n} \cdot \vec{\sigma}_{n}\right\}=1+3(-1)^{n},
$$

where the factor 3 stems from $\vec{\sigma}^{2}=3$. The same sequence of numbers $S_{n}=(-2,4,-2,4, \ldots)$ has been reported in section IV of ref.[5] without a derivation.

Next, we consider fermions with $g$ internal degrees of freedom. These "flavor" degrees of freedom are assumed to form the $g$-dimensional representation of the special unitary group $S U(g)$. The antisymmetrized contact-interaction which incorporates the Pauli exclusion-principle is now obtained by subtracting from the unit-operator $\mathbf{1}$ in two-particle flavor-space its Fierz transform:

$$
\mathbf{1}-\left(\frac{\mathbf{1}}{g}+\frac{1}{2} \vec{\lambda}_{1} \cdot \vec{\lambda}_{2}\right)
$$

Here, $\vec{\lambda}$ denotes $g^{2}-1$ traceless generators that are normalized as $\operatorname{tr}\left(\lambda^{a} \lambda^{b}\right)=2 \delta^{a b}$, with indices $a, b \in\left\{1, \ldots, g^{2}-1\right\}$. The operator $1 / g+\vec{\lambda}_{1} \cdot \vec{\lambda}_{2} / 2$ has indeed the properties of a flavor-exchange operator. Its eigenvalue in the $g(g+1) / 2$-dimensional representation of symmetric tensors is $1 / g+(g+2)(g-1) / g-\left(g^{2}-1\right) / g=1$, and its eigenvalue in the $g(g-1) / 2$-dimensional representation of antisymmetric tensors is $1 / g+(g-2)(g+1) / g-\left(g^{2}-1\right) / g=-1$. The starting expression for the spin-factor $S_{n}(g)$ reads:

$$
\begin{aligned}
S_{n}(g) & =\operatorname{tr}_{1} \operatorname{tr}_{2} \ldots \operatorname{tr}_{n}\left\{\left(\mathbf{1}-\frac{\mathbf{1}}{g}-\frac{1}{2} \vec{\lambda}_{n} \cdot \vec{\lambda}_{1}\right)\left(\mathbf{1}-\frac{\mathbf{1}}{g}-\frac{1}{2} \vec{\lambda}_{1} \cdot \vec{\lambda}_{2}\right) \ldots\left(\mathbf{1}-\frac{\mathbf{1}}{g}-\frac{1}{2} \vec{\lambda}_{n-1} \cdot \vec{\lambda}_{n}\right)\right\} \\
& =\operatorname{tr}_{2} \ldots \operatorname{tr}_{n}\left\{\left[g\left(\mathbf{1}-\frac{\mathbf{1}}{g}\right)^{2}+\frac{1}{2} \vec{\lambda}_{n} \cdot \vec{\lambda}_{2}\right] \ldots\left(\mathbf{1}-\frac{\mathbf{1}}{g}-\frac{1}{2} \vec{\lambda}_{n-1} \cdot \vec{\lambda}_{n}\right)\right\}
\end{aligned}
$$

and we have shown the intermediate result of a single flavor-trace, where $\operatorname{tr} \mathbf{1}=g$. After taking $n-1$ flavor-traces one arrives at:

$$
S_{n}(g)=\operatorname{tr}_{n}\left\{g^{n-1}\left(\mathbf{1}-\frac{\mathbf{1}}{g}\right)^{n}+(-1)^{n} \frac{1}{2} \vec{\lambda}_{n} \cdot \vec{\lambda}_{n}\right\}
$$

which leads with $\vec{\lambda} \cdot \vec{\lambda}=2(g-1 / g) \mathbf{1}$ to the final result:

$$
S_{n}(g)=(g-1)^{n}+(-1)^{n}\left(g^{2}-1\right) .
$$


One realizes that $g(g-1)$ is a common divisor of the polynomials $S_{n}(g)$ of degree $n$ in $g$. The spin-factors of the 3-, 4- and 5-ring diagrams are:

$$
\begin{aligned}
& S_{3}(g)=g(g-1)(g-3), \\
& S_{4}(g)=g(g-1)\left(g^{2}-3 g+4\right), \\
& S_{5}(g)=g(g-1)\left(g^{3}-4 g^{2}+6 g-5\right) .
\end{aligned}
$$

Although our derivation of the formula for $S_{n}(g)$ has been elementary, it seems to represent a novel result. We note as an aside that the same method applied to the symmetrized contact-interaction gives for bosonic $n$-ring diagrams the flavor-factor $B_{n}(g)=(g+1)^{2}+g^{2}-1=(-1)^{n} S_{n}(-g)$.

Let us finally generalize the expressions for the resummed interaction energy per particle in eqs. $(24,26)$ to arbitrary spin-degeneracy $g$. The sum of the leading $n$-ring diagrams with spinfactors $g^{n}$ takes the form:

$$
\bar{E}\left(k_{f}\right)_{\text {lead }}=\frac{k_{f}^{2} \alpha^{3}}{4 M}\left\{\frac{g^{2}}{4} G\left(\frac{\alpha g}{2}\right)\right\},
$$

and the complete ring-sum with inclusion of all exchange-type diagrams is given by:

$$
\bar{E}\left(k_{f}\right)=\frac{k_{f}^{2} \alpha^{3}}{4 M}\left\{\frac{(g-1)^{3}}{4 g} G\left(\frac{\alpha}{2}(g-1)\right)-\frac{g^{2}-1}{4 g} G\left(-\frac{\alpha}{2}\right)\right\},
$$

with the function $G(\alpha)$ defined in eq.(24) and displayed with its $\alpha$-dependence in Figs. 3,4.

\section{Acknowledgement}

I thank H.W. Hammer, T. Schäfer and R. Schmidt for informative discussions. This work has been supported in part by DFG and NSFC (CRC 110).

\section{References}

[1] M. Le Bellac, "Thermal Field Theory", Cambridge University Press, (1996).

[2] G. Baym and C. Pethick, "Landau Fermi-Liquid Theory", Wiley \& Sons, New York (1991).

[3] S. Babu and G.E. Brown, Ann. Phys. (NY) 78 (1973) 1.

[4] R.J. Furnstahl and H.W. Hammer, Ann. Phys. (NY) 302 (2002) 206.

[5] T. Schäfer, C.W. Kao and S.R. Cotanch, Nucl. Phys. A762 (2005) 82.

[6] H.W. Hammer and R.J. Furnstahl, Nucl. Phys. A678 (2000) 277.

[7] J.V. Steele, nucl-th/0010066; eq.(18) in that paper needs to be corrected to: $\bar{E}_{(2 d)}=-(g-1)\left(g^{2}-3 g+4\right)\left(k_{f}^{2} / 2 M\right)\left(a k_{f}\right)^{4} \cdot 0.00863945$.

[8] N. Kaiser, Eur. Phys. J. A49 (2013) 140.

[9] E.H. Hwang and S. Das Sarma, Phys. Rev. B75 (2007) 205418.

[10] B. Fröhlich et al., Phys. Rev. Lett. 106 (2011) 105301.

[11] M. Randeria, J.M. Duan and L.Y. Shieh, Phys. Rev. B41 (1990) 327.

[12] A.L. Fetter and J.D. Walecka, "Quantum Theory of Many-Particle Systems", McGraw-Hill, New York (1971); chapt. 12.

[13] F. Stern, Phys. Rev. Lett. 18 (1967) 546.

[14] B. Mihaila, cond-mat/1111.5337.

[15] E.K.U. Gross, E. Runge and O. Heinonen, "Many-Particle Theory", IOP Publishing Ltd, Bristol (1991); chapt. 22. 